\title{
Diagrama de Voronoi e Eixo Medial CNMAC 2014
}

\author{
Eneas Mendes de Jesus \\ Bolsista do Programa de Educação Tutorial FNDE, PETMAT-UFES \\ E-mail: neo.mdj@gmail.com
}

Fabiano Petronetto do Carmo

Departamento de Matemática - Universidade Federal do Espirito Santo

E-mail: fabiano.carmo@ufes.br.

\section{$\underline{\text { RESUMO }}$}

O eixo medial é um conceito geométrico que corresponde aos pontos localmente simétricos de uma figura e é baseado em objetos contínuos e curvas fechadas simples, ao passo que o diagrama de Voronoi é obtido a partir de um conjunto de pontos discretos. Neste trabalho, definiremos tais conceitos geométricos de tal forma a evitar a inconsistência contínuo-discreto dada a partir das definições clássicas destes conceitos, podendo então obter o eixo medial a partir do diagrama de Voronoi para uma gama de objetos discretos incluindo aproximações de curvas com auto-interseção e curvas abertas.

Diagrama de Voronoi é um conceito geométrico baseado na distância a pontos de um dado conjunto discreto. Seja $P=\left\{p_{1}, p_{2}, \ldots, p_{n}\right\}$ um conjunto de pontos no $\mathbb{R}^{n}$. O Diagrama de Voronoi de $\mathrm{P}$, denotado por $\mathcal{D} \mathcal{V}(P)$, é a subdivisão do plano em regiões, onde cada região, chamada Região de Voronoi e denotada por $\mathcal{R} \mathcal{V}\left(p_{i}\right)$, consiste de todos os pontos que estão, pelo menos, mais próximo a $p_{i}$ que qualquer outro ponto de $P$. Tem-se $\mathcal{R} \mathcal{V}\left(p_{i}\right)=\left\{x \in \mathbb{R}^{n} ; d\left(x, p_{i}\right) \leq d\left(x, p_{j}\right), \forall p_{j} \in P \operatorname{com} j \neq i\right\}$.

Esta definição de diagrama de Voronoi não é aplicável a objetos contínuos (Figura 1). Na ilustração superior, o site $^{1} e_{2}$ é um segmento de reta (contínuo), enquanto na inferior, o segmento é dado por um conjunto infinito de pontos $e_{t}$. Observe que as figuras tem Diagramas de Voronoi diferentes.

Dado o diagrama de Voronoi $\mathcal{D} \mathcal{V}(P)$ de um conjunto de pontos $P \in \mathbb{R}^{n}$ podemos definir o dual de $\mathcal{D} \mathcal{V}(P)$ como sendo a estrutura obtida a partir de $\mathcal{D} \mathcal{V}(P)$ ligando os pontos de $P$ que compartilham de uma mesma aresta.

Dado um conjunto de pontos $P \in \mathbb{R}^{n}$ (não todos colineares) definimos a triangulação de Delaunay, denotada por $\mathcal{T} \mathcal{D}(P)$, como sendo o dual de $\mathcal{D} \mathcal{V}(P)$. A triangulação de Delaunay é a "melhor triangulação" de um conjunto de pontos ${ }^{2}$. A unicidade é garantida observando-se algumas restrições, por exemplo, em $\mathbb{R}^{2}$ é necessário que não haja em $P$ quatro pontos em uma mesma circunferência.

Outra estrutura geométrica bastante conhecida é o eixo medial. $\mathrm{O}$ eixo medial é em geral definido a partir de bolas maximais. Considere $S$ uma curva fechada em $\mathbb{R}^{n}$. Uma bola aberta interior a $S$ que não

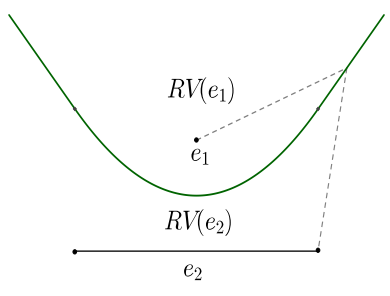
está contida em nenhuma outra bola aberta interna à $S$ é chamada de

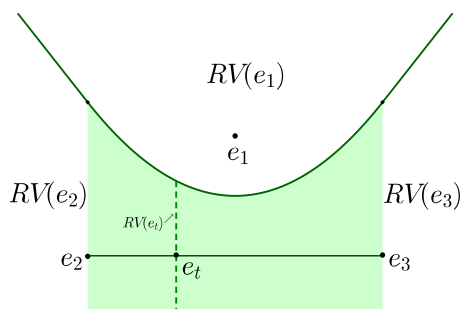

Figura 1: Diagrama de Voronoi.

\footnotetext{
${ }^{1}$ site: um objeto geométrico contido no seu conjunto

${ }^{2}$ A Triangulação de Delaunay maximiza o menor ângulo de todos os triângulos na triangulação; esta tende a evitar triângulos com ângulos internos muito pequenos.
} 
bola maximal. O eixo medial é definido como o lugar geométrico dos centros de todas as suas bolas maximais. Para tal definição é necessário que o interior de $S$ esteja bem definido, excluindo assim a possibilidade de aplicação a objetos discretos e curvas abertas ou com auto-interseção.

Neste trabalho, estudamos uma relação entre o diagrama de Voronoi e o eixo medial de tal forma a obter o eixo medial a partir do diagrama de Voronoi de maneira semelhante a construção da triangulação de Delaunay. Apresentamos pequenas modificações nas definições de ambos os conceitos, mantendo as boas propriedades já existentes das definições clássicas. Estendemos o diagrama de Voronoi a objetos contínuos, tais como curvas abertas, fechadas e com auto-interseção, e em contra partida estendemos o conceito de eixo medial a objetos discretos e curvas abertas e com auto-interseção.

Como já ilustrado na figura anterior, considerando um segmento de reta $A B$ qualquer em $\mathbb{R}^{2}$ como sendo um conjunto infinito de pontos, a região de Voronoi de um ponto $e \in A B$ será a reta normal a $A B$ que passa por $e$. Mas, se considerarmos $A B$ como sendo um único site, então tais retas não farão parte do diagrama de Voronoi. A definição clássica de região de Voronoi não nos permite a aplicação do diagrama de Voronoi a objetos contínuos. Por outro lado, para o eixo medial a definição clássica nem se aplica a objetos discretos devido a definição de bola maximal.

A seguir, dado $E \subset \mathbb{R}^{n}$ um conjunto de sites, definimos tais conceitos geométricos tornando possível obter o diagrama de Voronoi de forma consistente para objetos contínuos e, da mesma forma, o eixo medial para objetos discretos. Além disso, obtemos uma relação entre os conceitos apresentados.

A região de Voronoi de um site $e \in E$, é o conjunto de pontos estritamente mais próximos a $e$ que qualquer outro site em $E$. A partir daí, definimos o diagrama de Voronoi como sendo todos os pontos de $\mathbb{R}^{n}$ que não pertencem à nenhuma das regiões de Voronoi.

Uma bola aberta $B$ é dita maximal se $B$ não possui nenhum ponto de $E$ e $B$ não está contida propriamente em nenhuma outra bola aberta com esta propriedade, não sendo mais necessário que $E$ tenha seu interior definido. O eixo medial é o lugar geométrico dos centros de todas bolas maximais cuja fronteira tem mais que dois pontos de interseção com $\mathrm{S}$.

Temos agora definido dois conceitos que atuam sobre o mesmo objeto cuja a relação é apresentada neste trabalho de forma natural a partir das definições. Para formalizar e identificar sob quais condições estes dois conceitos são relacionados, dois principais resultados são demonstrados neste trabalho.

A Figura 2 ilustra o diagrama de Voronoi para conjuntos discretos de pontos sobre um retângulo. Em verde, destaca-se o eixo medial do retângulo (contínuo). Na esquerda, notamos que os centros das bolas maximais coincidem com os pontos que estão sobre o diagrama de Voronoi, e na direita observamos que ao aumentar a quantidade de pontos discretos sobre o retângulo, parte do diagrama de Voronoi se aproxima do eixo medial do retângulo, sendo esse mais um importante resultado obtido neste trabalho.
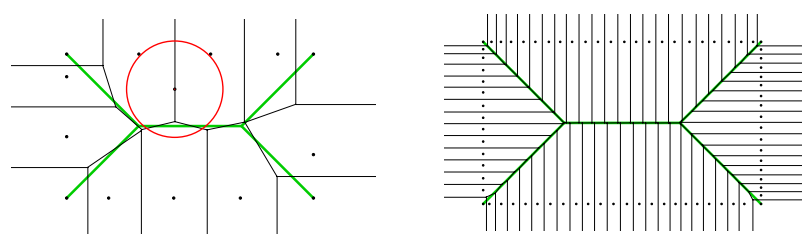

Figura 2: Diagrama de Voronoi e Eixo Medial.

\section{Palavras-chave: Geometria Computacional, Diagrama Voronoi, Eixo Medial. Referências}

[1] J.o’Rourke. Computational geometry in C. Cambridge university press, 1994.

[2] L.H.Figueiredo, e P.C.Carvalho. Introdução a geometria computacional. $18^{\circ}$ Colóquio Brasileiro de Matemática - IMPA. 1991.

[3] M. De Berg, M. Van Kreveld, M. Overmars, O. Scwarzkopf, Computational geometry. Springer 1997.

[4] R. Fabri, L.F.Estrozi e L.F.Costa. On Voronoi Diagrams and Medial Axes. Journal of Mathematical Imaging and Vision, 2002,17(1), 27-40. 